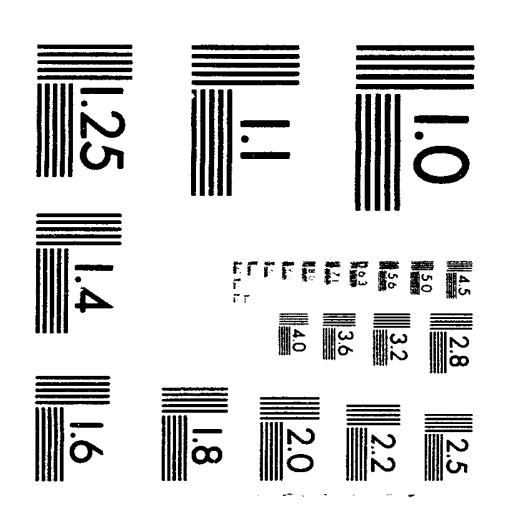



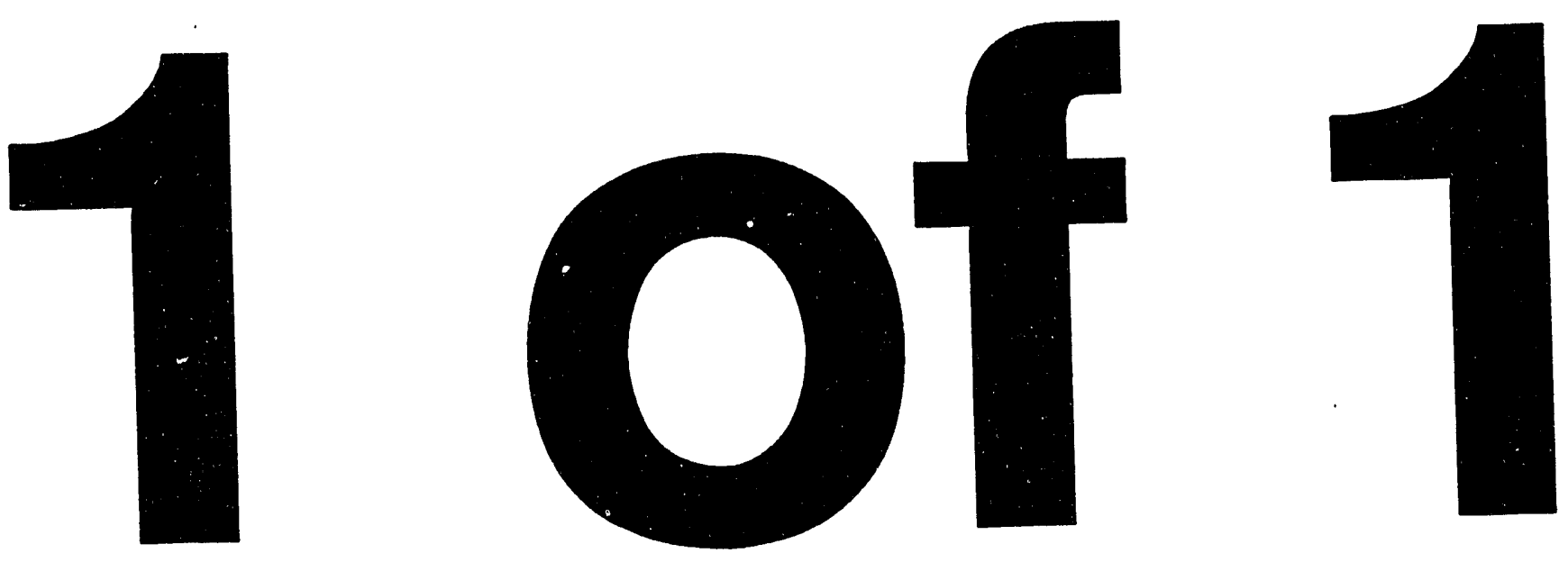


\title{
DISCOVERY AS A PROCESS
}

\author{
Craig Loehle \\ Environmental Research Division \\ Argonne National Laboratory \\ 9700 S. Cass Avenue \\ Argonne, IL 60439
}

\section{DISCLAIMER}

This report was prepared as an account of work sponsored by an agency of the United States Government. Neither the United States Government nor any agency thereof, nor any of their employees, makes any warranty, express or implied, or assumes any legal liability or responsibility for the accuracy, completeness, or usefulness of any information, apparatus, product, or process disclosed, or represents that its use would not infringe privately owned rights. Reference herein to any specific commercial product, process, or service by trade name, trademark, manufacturer, or otherwise does not necessarily constitute or inply its endorsement, recommendation, or favoring by the United States Government or any agency thereof. The views and opinions of authors expressed herein do not necessarily state or reflect those of the United States Government or any agency thereof.

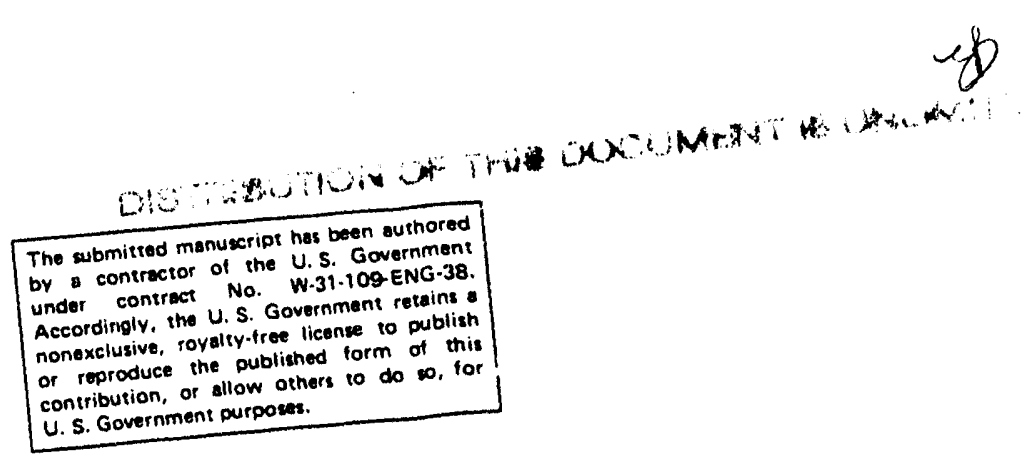




\section{INTRODUCTION}

Scientific discovery is one of the most dramatic and exciting products of the human mind and ultimately is the source of our advanced technology. There is tremendous pressure to increase the rate of discovery as more and more key societal issues contain a scientific component (e.g., AIDS, global climate change, tropical deforestation). Currently expectations are very high because of science's past breakthroughs, but the general perception of how science works is not in harmony with its actual operation. This discrepancy leads to the attitudes reflected in AIDS protests that the government is not doing enough, even though over $\$ 1$ billion is being spent annually on research, which is more per AIDS mortality case than is spent for any other disease. The expectation that money alone is required to order a cure on demand results from a serious misunderstanding of the discovery process.

To enhance scientific discovery, funding is of course necessary, but so is a clearer understanding of how discoveries are actually made, so that barriers to discovery can be removed and discoveries can be rewarded and enhanced. As Bauer (1992) pointed out, science is not based on a fixed recipe or method (the "scientific method"). If it was, we would not be frustrated by problems such as cancer, and scientific progress would be predictable. Rather, science proceeds by a process similar to solving a puzzle. That is, discovery is a process, not an event, and in fact is a rather complicated process. The official version of how science is or should be done conflicts with this reality.

Some persistent myths about scientific discovery cause a great deal of trouble and confusion in the practice and public perception of science today. 
The three great myths, which form a sort of triumvirate of misunderstanding, are the Eureka! myth, the hypothesis myth, and the measurement myth. These myths are prevalent among scientists as well as among observers of science. The Eureka! myth asserts that discovery occurs as a flash of insight, and as such is not subject to investigation. This leads to the perception that discovery or deriving a hypothesis is a moment or event rather than a process. Events are singular and not subject to description. The hypothesis myth asserts that proper science is motivated by testing hypotheses, and that if something is not experimentally testable then it is not scientific. This myth leads to absurd posturing by some workers conducting empirical descriptive studies, who dress up their study with a "hypothesis" to obtain funding or get it published. Methods papers are often rejected because they do not address a specific scientific problem. The fact is that many of the great breakthroughs in science involve methods and not hypotheses (e.g., the electron microscope, X-ray diffraction methods, PCR technology, Hall, 1992; Crease, 1992) or arise from largely descriptive studies. Those captured by this myth also try to block funding for those developing methods. The third myth is the measurement myth, which holds that determining what to measure is straightforward, so one doesn't need a lot of introspection to do science. As one ecologist put it to me "Don't give me any of that philosophy junk, just let me out in the field. I know what to measure."

These myths lead to difficulties for scientists who must face peer review to obtain funding and to get published (Bauer, 1992). These myths also inhibit the study of science as a process. Finally, these myths inhibit creativity and suppress innovation. In this paper I first explore these myths in more detail 
and then propose a new model of discovery that opens the supposedly miraculous process of discovery to closer scrutiny.

\section{Myth 1: The Eureka! Myth}

It has long been assumed that the philosophy of science may be helpful in epistemology or in the logic of deduction, axiomatization, or justification, but that it has little to say concerning the process of discovery. Popper (1963), for instance, stated that discovery (the generation of hypotheses) is not subject to formal rules, so that neither inductive nor deductive logic are reliable (though they are sometimes useful) paths to discovery. This idea has led to the view that scientific discovery is somehow a mysterious subconscious process, a conclusion that does not necessarily follow from Popper's argument. Many have written about flashes of insight, dreams, the creative personality, etc. (e.g., Root-Bernstein, 1989). In this view, discoveries appear as a creative act, in a flash. If one is not a "creative type," then one won't have creative flashes.

The view that discoveries, insights, or hypotheses are obtained in a flash is what I call the Eureka! myth. It is reinforced by dramatic accounts like Kekule's discovery of chemical ring structures in a dream and similar accounts (c.f., Hadamard, 1945). Conscious work - incubation - insight has in fact become the official model of discovery in the creativity literature. Such colorful, flash-of-insight accounts capture the imagination and are certainly interesting, but they may be misleading. They draw attention to only the dramatic cases and suggest that the process of discovery is not subject to study or dissection. 
In contrast to this Eureka! view, I would like to argue for the central role of pattern recognition in the discovery process. The human brain is wired for pattern recognition, as Margolis (1987) has argued at length. This process is neither inductive nor deductive, nor is it rule-based, though it can be mimicked by rules in some cases. For example, everyone can recognize, at a flash, hundreds if not thousands of faces, without using statistical hypothesis testing or deductive logic. Many people are equally good at musical patterns, being able to recognize thousands or even tens of thousands of songs. They generally would be unable to do so from the sheet music and absolutely unable to do so from a sonogram. This raises an interesting question: If all we had was the sonogram of a sound, would we be likely to ascribe meaning or significance to it? See Fig. 1 for an illustration of the difficulty. One colleague described Fig. 1 as "some rhythmic pattern, maybe a pressure wave" whereas another (who knew it was a sonogram) thought it was probably frog or cricket calls.

In addition to visual and auditory patterns, some people have a facility for recognizing other, more abstract types of patterns. Einstein described his own thought processes as being of this type (Hadamard, 1945). Such a skill is particularly useful for finding relationships in phenomena that do not necessarily have a visual representation.

There is a crucial difference between detecting patterns in science and the popular concept of creativity. Pattern recognition is not a free, uninhibited, joyous, artistic type process. It is far closer to puzzle solving or mechanical work. That is, a pattern or mental structure or understanding does not 


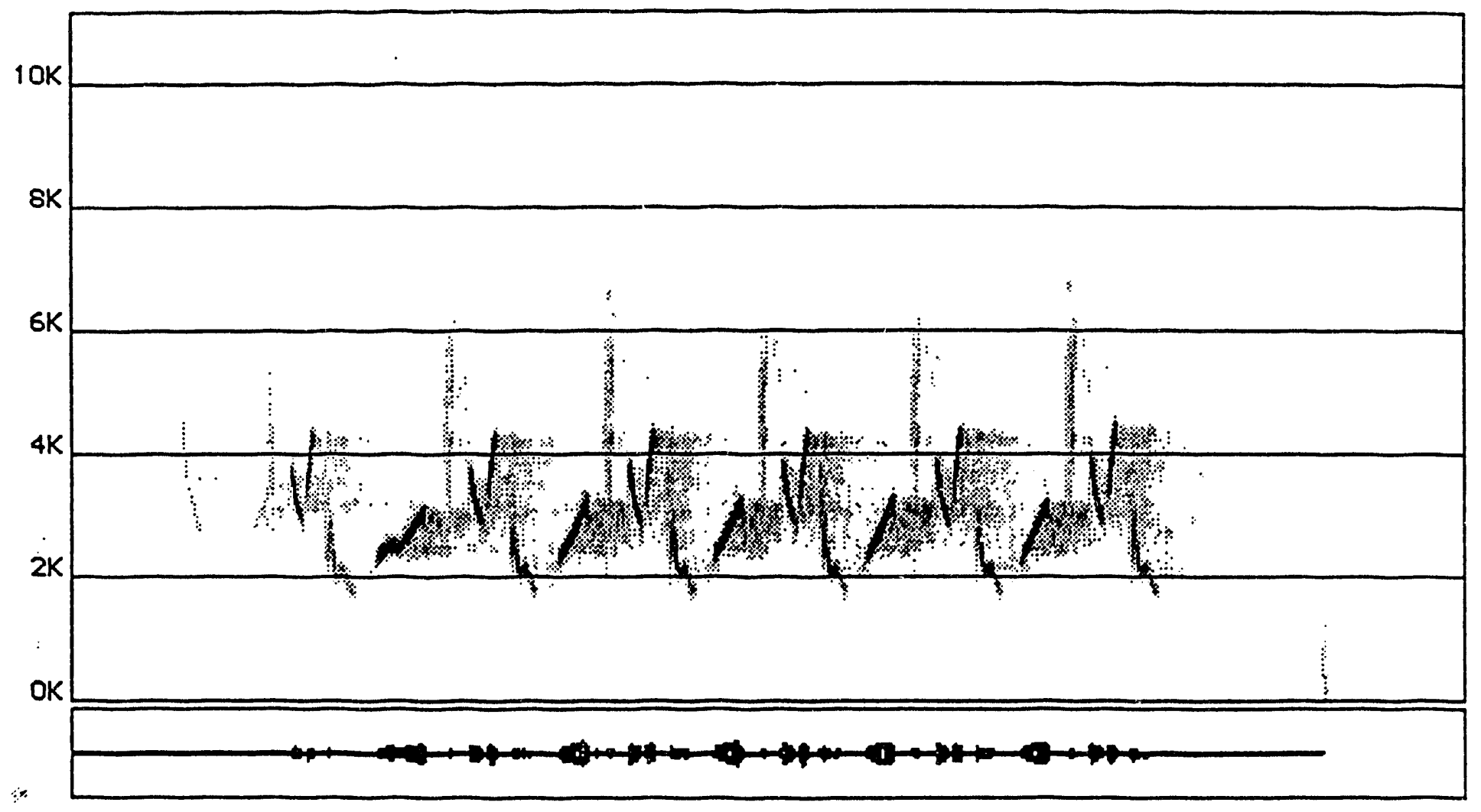

Figure 1. Acoustic signature (sonogram and waveform diagram) of the Carolina Wren. Units are $\mathrm{kHz}$. Very few people can recognize that this pattern is birdsong. Figure provided by Carey Tuckfield. 
necessarily come all as a piece and in a flash, but it may be built up slowly and piecemeal as one links facts together and builds and rearranges a mental framework for the problem. It involves tinkering, puttering, patience, and stubbornness. That is, we may say that the scientist is involved in constructing patterns. These patterns consist of networks of relationships between facts, assumptions, mathematical relations and methods, measurement techniques, rules of thumb, and hunches. A "discovery" involves an expansion, rearrangement, or simplification of all or part of this network of relationships. A discovery is thus not a thing or event and rarely involves only a single step.

Myth 2: The Hypothesis Myth

It has become a truism that one can not design a proper experiment without a clearly stated hypothesis. To an extent this is a reasonable approach as a response to the pure empiricist tradition, because the mere gathering and cataloging of "facts", the Baconian method, only leads to a very large pile of facts and very little knowledge. The emphasis on hypotheses may be traced to Popper's (1963) Principle of Demarcation, which gives testability as the demarcation between scientific and unscientific (e.g., astrology, Marxism) theories. This perfectly correct argument has been carried to too fine a level of detail, however, by the general scientific establishment.

The argument that a theory must be testable has been extended to the requirement that every aspect of science must involve a theory (hypothesis) and a test of that theory (hypothesis). This narrow interpretation of "testability" is blind to the fact that much of science is really technology. 
Scientists spend much of their time developing instruments, software, and methods to measure and detect phenomena (Crease, 1992; Hall, 1992). This activity is science, but it is not a theory nor a test of a theory. In addition, empirical, descriptive studies are a necessary part of science. It is from them that sufficient data may be obtained to allow a pattern (structure, relationship) to be detected, possibly forming the basis for further study. Such preliminary observed patterns are not hypotheses in the sense of Popper because they are empirical rather than explanatory.

We may trace this confusion over what is a hypothesis partially to the overly simplified presentations of the scientific method taught in school and partially to the confounding of concepts between a scientific hypothesis and a statistical hypothesis. A statistical hypothesis is a probability statement in terms of outcomes. It concerns measurable events and magnitudes. In contrast, we may have a perfectly valid scientific hypothesis (theory) but be unable to specify its outcomes. A deductive step is required to go from the scientific hypothesis to the statistical hypothesis, and this step is not always easy or clear cut. Stating a statistical hypothesis as though it were a scientific hypothesis gives the appearance of trying to buy respectability. If we conduct a purely empirical study of the efficacy of a pesticide (with no biochemical analysis of how it works) by measuring dose versus kill rate, we have no scientific hypothesis, but we do have a statistical hypothesis framed in terms of the experimental design. Studies that quantify or compare are valid in their own right and need not be embellished to justify them.

A final source of confusion arises from multiple definitions of "scientific hypothesis." If I say "I think $X$ and $Y$ may be related in some way," this is a 
hunch or intuitive guess. This is what we have when we think we have found a regularity (pattern) in nature. Comparing $X$ and $Y$ does not "test" this guess because being wrong on such a hunch or "working hypothesis" does not cause any change in a scientific paradigm. In contrast, a scientific hypothesis is based on cause-effect reasoning. A scientific hypothesis does not merely state that $X$ and $Y$ may be related, but it explains why they are related. Should we find that $X$ and $Y$ are not related when a theory predicts them to be, then something is wrong with the theory. If we have a hunch that they are related, but then find that they are not, we merely try something else. The correlation between body size and lifespan in mammals is not a hypothesis or a theory, but any potential explanations for this correlation are.

The essential point about hypotheses is that there are different types and levels of hypothesis. At the first stage of discovery we have a hunch or intuition that we have detected a pattern or relationship in nature. This working hypothesis is a perfectly valid subject of study. A working hypothesis may lead to the discovery of an empirical relationship which may be quite precise but is not a theory. A working hypothesis becumes a scientific hypothesis or theory when one offers an explanation for a pattern or relationship in terms of cause-effect or structural properties. A statistical hypothesis may be purely empirical (dog food brand A is better), or descriptive (a quadratic line fits these data), or it may be used to test a scientific hypothesis after suitable deduction and definition of variables. 


\section{Myth 3: The Measurement Myth}

The measurement myth is the assumption that defining variables to measure for statistical testing is a straightforward matter. Pattern recognition often violates the statistical assumption that the objects to be measured are well defined. This fact becomes clear when we examine pattern recognition in some everyday contexts. It is not at all obvious what features we use for recognizing a familiar face. We never measure and plot facial features to identify people and would nor even be able to say what variables are informative nor how to measure them. (How do you quantify "beautiful eyes"?) Furthermore, we have no clue about how we are processing this information. Such a process is "unscientific" because it has no hypothesis and no mechanism, and yet it is reliable.

We observe that as a discovery unfolds and is elaborated, all of the primitives (facts, metrics, variables, objects) may be called into question. The solid atom may turn out to be composed of parts. Solid continents may turn out to move. Rulers may shrink and grow and clocks nove at different speeds. Thus, the basic assumption of statistics and experimental design that the variables to be measured are well defined and quantifiable does not hold up in the early stages of scientific discovery. This argument demonstrates that the step from hypothesis to experiment is not necessarily straightforward.

\section{A New Model}

I would like to propose an alternative to the standard model of the scientific method. My model emphasizes pattern and its elaboration as being 
prior to formal statistics and hypothesis testing. The four steps are the following:

1. A pattern is found, a defect in an existing pattern is found, or no pattern is found where one was expected. The pattern may be in data, or it may be a meta-pattern, relating abstract concepts.

2. Elaboration takes place. A vocabulary must often be developed to describe the pattern. Methods of measurement (of shape or form, statistical properties, instruments, laboratory protocol) may need to be developed. Characteristics of the pattern are refined.

3. A theory or explanation for the observed pattern is proposed. The theory may need elaboration, rearrangement, or analysis before it yields testable predictions.

4. Experimental, statistical testing of hypotheses begins.

The detection of pattern at stage 1 is what is usually meant by "discovery." During the conduct of routine science, one can often move rapidly from stage 1 to stage 4, and the processes of stages 2 and 3 tend to be glossed over or lost in the final scientific report of a study. In many cases, however, the real work of discovery takes place iteratively at stages 1, 2, and 3, but differs in nature and is often done by different people at different stages. In certain disciplines, the process of pattern definition and elaboration turns out to be painfully prolonged and is thus subject to closer examination. Two such cases are explored below. 
In the initial stages of pattern detection, it may be instantly clear that a pattern has been found but not at all clear how to proceed next. Strange attractors were not well received at first when expressed mathematically (Gleick, 1987a), but when they were presented visually it was recognized instantly that real patterns were involved. For example, the sequence in time of water dripping from a faucet appears random, but when the dripping is plotted as an attractor (Gleick, 1987b), the pattern becomes apparent. Once attractors were recognized from graphic plots, it became clear that a descriptive vocabulary was needed. For example, the attractor for a pendulum (Fig. 2) is clearly not random, but is very difficult to describe. Statistically demonstrating the difference between random and chaotic time series is quite difficult and new methods have been needed. The initial intuitive recognition of attractors has thus been augmented by the development of tools such as the concepts of scaling and universality and the use of Poincare' sections.

We might also recognize patterns via the Gestalt process of figureground reversal. For example, we might fail to see a consistent pattern in locations where a species is found but see a pattern in locations where the species is not found. As another example, one might study not only the attractor of a dynamic system but also its repellor, which may provide new insights (Sidorowich, 1992). We might make a discovery by observing the lack of a pattern or response where one was expected. Such was the case in the discovery of symmetry breaking in physics. Finally, we may make a discovery by observing a defect in a pattern, an anomaly (Lightman and Gingerich, 1991). Such was the origin of relativity: a defect, largely ignored, in Newtonian mechanics. 

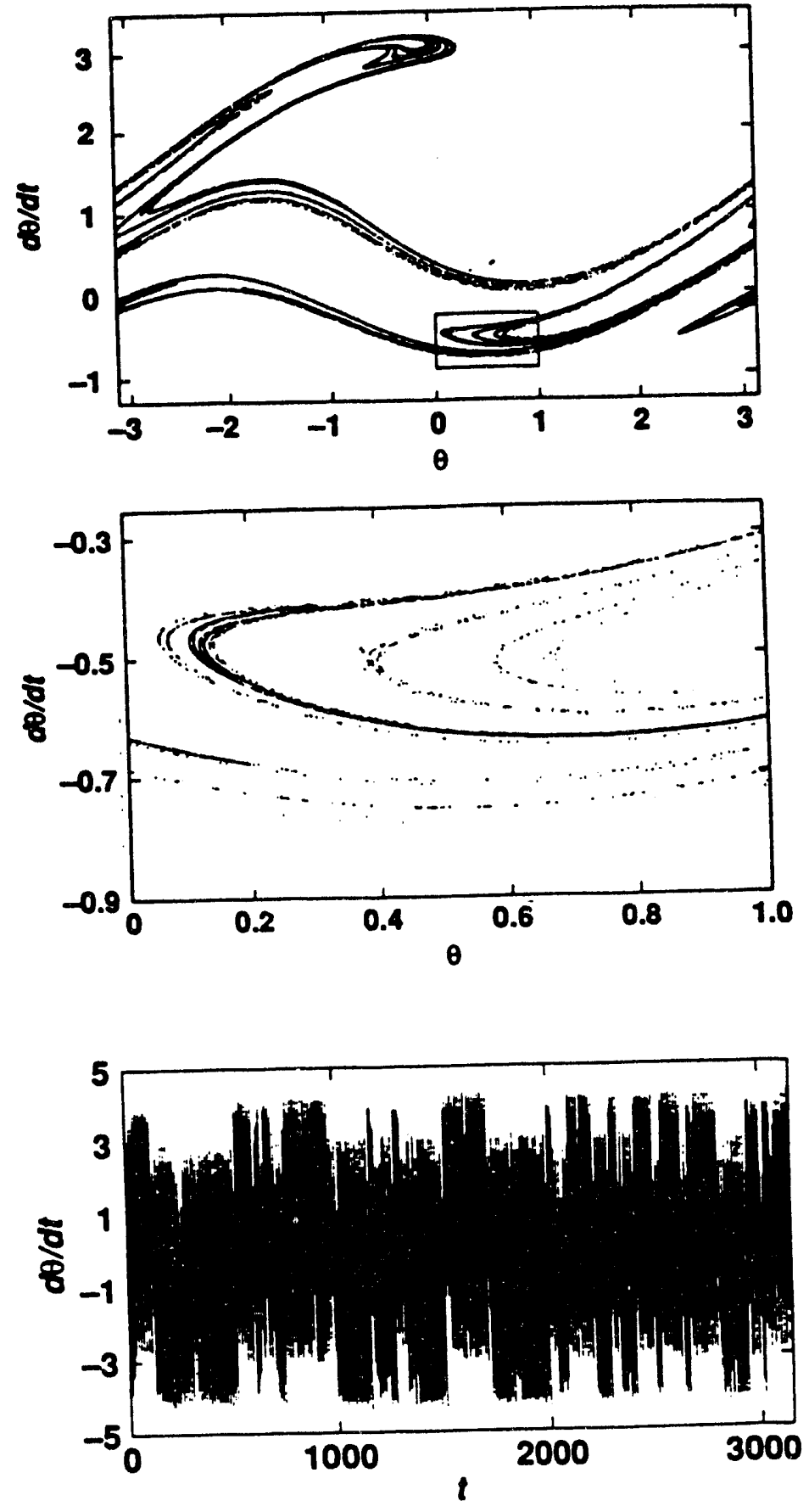

Figure 2. Dynamics and attractor for a pendulum (from Grebogi et al., 1987, Fig. 5 and 6). 
The process of pattern elaboration is central to the study of patterns for which cause and effect cannot be discerned immediately by experimentation. During the elaboration stage, the problem of how and what to measure is crucial. For example, in the early X-ray diffraction studies of DNA (Watson, 1968) what was being measured and how to interpret the data were not at all clear. What is foreground and what background, what is data and what noise, all need to be worked out. In addition, a vocabulary for description is essential. The trained taxonomist need only glance at most trees to identify them, but in doubtful cases he must refer to a key that uses a specialized descriptive vocabulary for leaf shape, flower parts, etc. Much of mathematics serves to provide a descriptive vocabulary. The process of developing a vocabulary in itself may change the perceived pattern. For example, in describing body form in the context of taxonomy, it was found that axis of symmetry was a useful descriptor. Radial symmetry is characteristic of simpler, more primitive forms (such as sea urchins) compared to the bilateral symmetry exhibited by all vertebrates. The recognition of bilateral symmetry led to the question of symmetry breaking such as the handedness of the large claw in crabs or right-hand dominance in humans. Thus the process of elaboration for both description and measurement may change our perception of the original pattern or uncover further patterns, all without necessarily involving hypotheses, explanations, or statistical tests. Further aspects of pattern elaboration are discussed in Loehle (1988).

By this account, several aspects of the conduct of science need rethinking. First, philosophers who view science as a branch of formal logic can be seen to be grossly mistaken. Such formal treatments as axiomatization of a theory 
become possible only long after the discovery phase. This is why practicing scientists are interested in historical accounts of iscoveries but give formal philosophical treatments little consideration. Second, the recognition that the elaboration phase is often a crucial step in the discovery process should help balance funding imbalances. Because the idea is ingrained that the business of science is doing experiments and "testing hypotheses", the vast majority of funding goes to large-scale data collection and experimental work, and only a pittance to elaborative research. As Stanford biologist David Botstein noted (Hall, 1992) with respect to biomedical research, "people are uncomfortable with research projects designed to improve technology as opposed to a research project designed to extract a few facts." For example, tens of millions of dollars are going toward construction and launching of satellites for data collection on earth systems such as EOS, and almost nothing on methods for using and interpreting the data that will be collected. As another example, a colleague requested $\$ 30,000$ to reanalyze, in the light of current theory, ecological field data he had collected 20 years previously. The agency replied that it does not fund researchers to "write papers," which should have been done on the original grant, but it would be happy to give him $\$ 130,000$ to do another field study! Elaboration and methods development are key components of the discovery process and should be given proper emphasis. In the absence of elaboration, discoveries remain only hunches or fail to be converted into widely used, reliable techniques (Crease, 1992). 


\section{CASE STUDIES}

Two cases from ecology are explored here to show how pattern recognition and elaboration may occur. The first, tree branching morphology, shows (1) how a single set of real physical objects can lead to the "recognition" of different patterns and (2) that an object, relationship, or fact in one context is not necessarily an object, relationship, or fact in another context. The second, food web structure, illustrates that measurement of an apparently straightforward pattern can be seriously confounded and obscure in practice.

\section{Tree Branching Morphology}

Different trees have different crown shapes and branching patterns. Indeed, different species can often be distinguished solely from their leafless profiles. These species differences may have adaptive significance, but their study has been hampered by the absence of a descriptive vocabulary or a way to organize the information. This dilemma is very similar to the problem of recognizing faces. Over time, various patterns have been observed and classification schemes developed for trees. A comparison of these various schemes reveals that they are not theories, being without explanatory content, and that what is a fact or measurable unit or discrete object differs from one proposed scheme to another.

One scheme involves classification of branching order based on a scheme previously applied to stream and river systems. When stream systems are classified, the smallest streams are of order 1 . Where two firstorder streams join, the stream below this point is of order 2, etc. When it is 

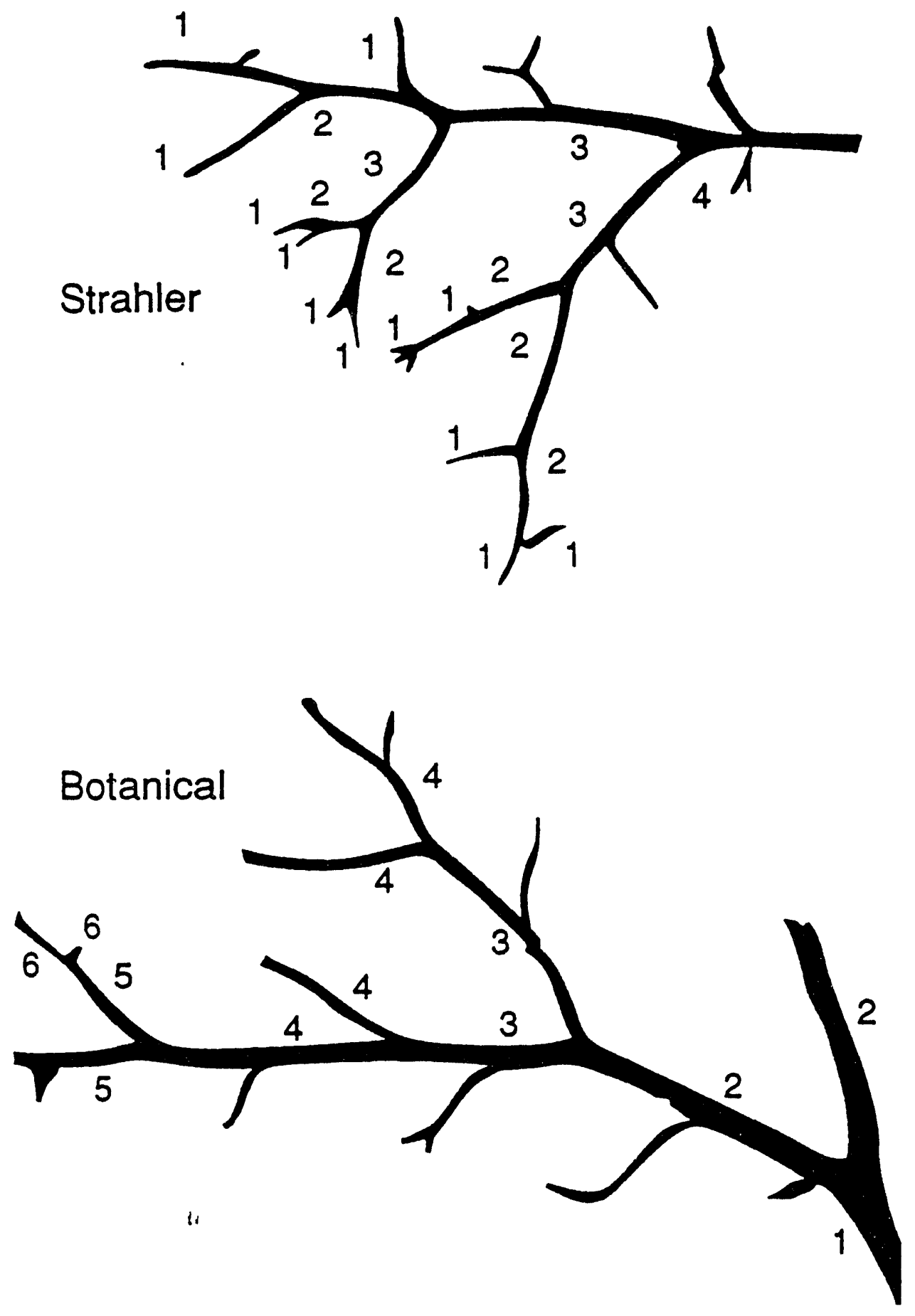

Figure 3. Branch ordering by position in the Strahler and botanical schemes. (Redrawn from Borchert and Slade, 1981). 
applied to tree branches, this scheme is referred to as the Strahler system (Borchert and Slade, 1981). Because numbering branches in the reverse order of their growth seems unnatural, the botanical numbering system counts branch order outward from the stem (Borchert and Slade, 1981). Fig. 3 illustrates both ordering schemes. The Strahler system has the advantage that all the terminal branches, where the leaves are, are of the same order and thus are comparable photosynthetic units, whereas the botanical system seems to reflect growth history. A third scheme (Fig. 4) reflects branch age rather than branch order (Agu and Yokoi, 1985). Branch age gives a very different picture than does branch order, but it is hard to measure on older trees. A fourth scheme is somewhat more abstract. Imagine cutting a tree into segments of equal length and then sorting the segments into diameter classes. The result is a regular pattern of decreasing frequency with diameter on a log-log plot (Shinozaki et al., 1964), as shown in Fig. 5. In this fourth scheme, the regularity of form takes no account of branch age or branch order, but only branch size. The three schemes that calculate branch order are topologically oriented, whereas the frequency-diameter relation is somewhat more abstract. Thus, these four patterns emphasize different features and require different measurement schemes.

My purpose here is not to say which of these schemes is "best" but rather to point out that progress in the study of tree branching requires that some pattern be found and that methods of measurement be defined. No a priori "facts" or defined entities could be said to exist in any of these cases before the pattern was defined. Pattern elaboration must precede any theorizing or experimentation regarding the adaptive significance or hormonal control of growth. 


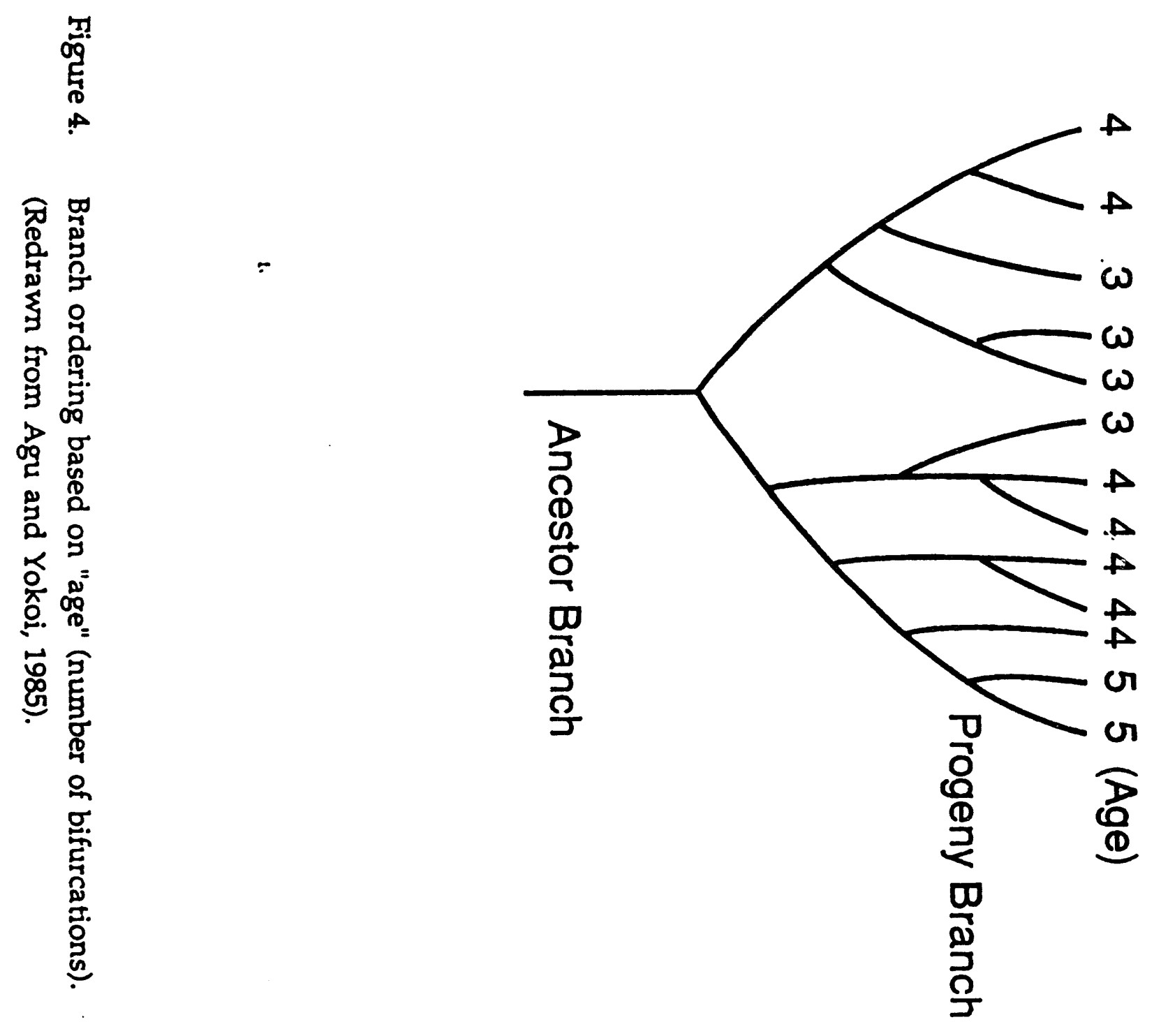




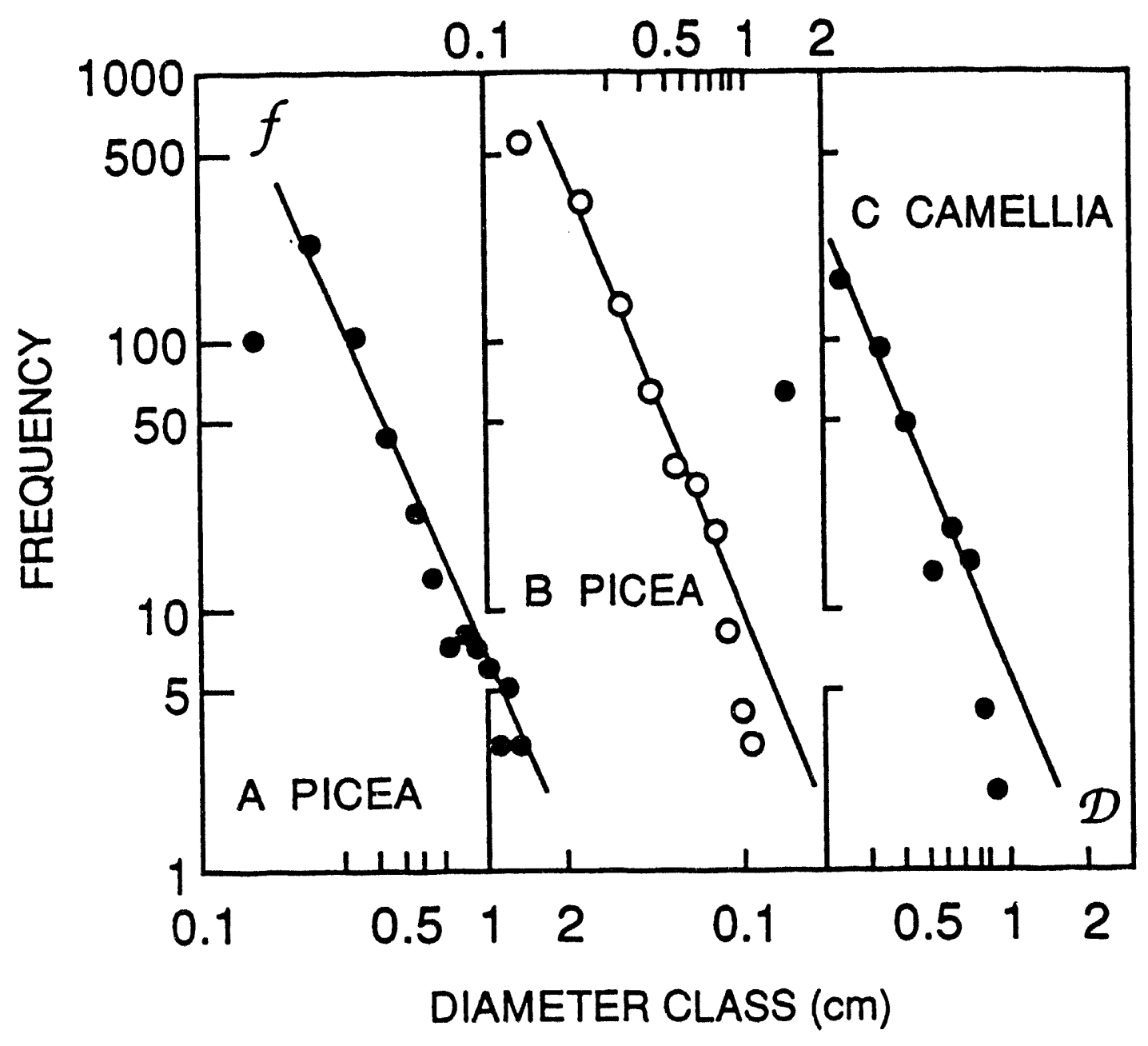

$1 ;$

Figure 5. Frequency distribution of diameters of branches for A) Picea jezoensis, B) P. Glehni, C) Camellia japonica. (From Shinozaki et al., 1964). 


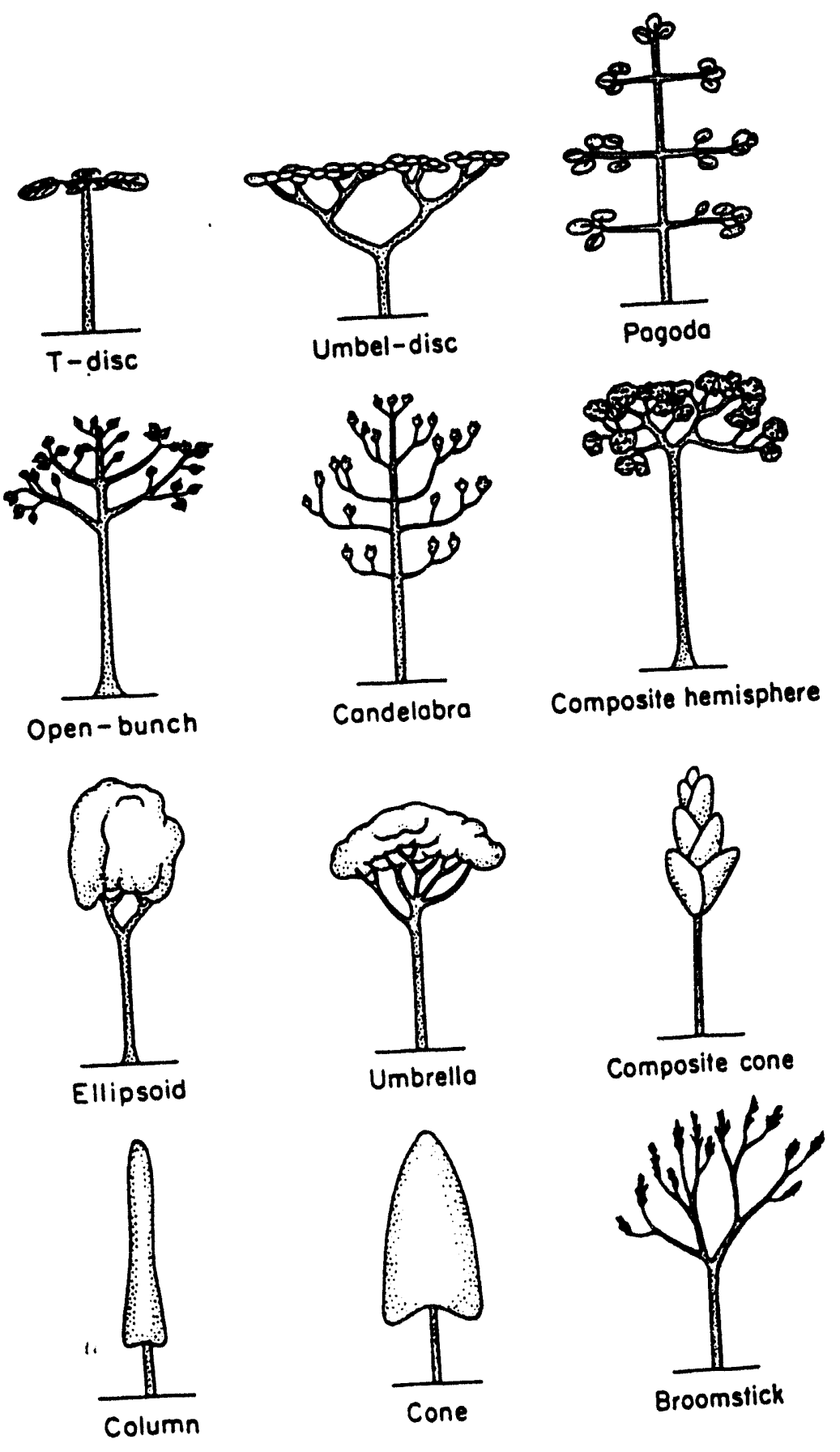

Figure 6. Major crown-shape ideotypes of Brunig (1976). (From Waller and Steingraeber, 1985). 
A second major approach to tree morphology emphasizes the entire tree shape. Various investigators over the years have distinguished certain characteristic tree shapes. Their work has led to various classification schemes, one of which is shown in Fig. 6. In this scheme the details of branch order are not important, nor are characteristics of the tree such as opposite versus alternate branching, leaves versus needles, etc. This proposed pattern has little theoretical content and is not explanatory, but it does form a basis for investigating ecological relations. We can see whether any of the proposed growth form types are consistently found in a particular habitat or exhibited by a particular taxonomic class (e.g., conifers). This scheme is not based on any theory and is thus not necessarily internally consistent or complete. That is, unlike the branch order scheme, the different ideotypes are not based on a single classification rule. Given the biologically intuitive basis for derivation of these ideal types, however, one may expect some ecological significance to be attributable to at least some of these different growth forms.

An alternate classification based more closely on a single set of classification rules is shown in Fig. 7. This scheme is based on the fate of terminal branches and the influence of flowering on branching pattern. For example, in some trees terminal flowering causes repeated dichotomous branching, strongly determining overall form. This classification scheme is much closer to being a theory rather than merely a raw pattern, because it contains some explanatory content. It does not a priori explain why certain patterns are found, but it does help to show how the patterns originate developmentally. In contrast to the branch-ordering schemes described above, these tree architecture models recognize basic units (variables in the 


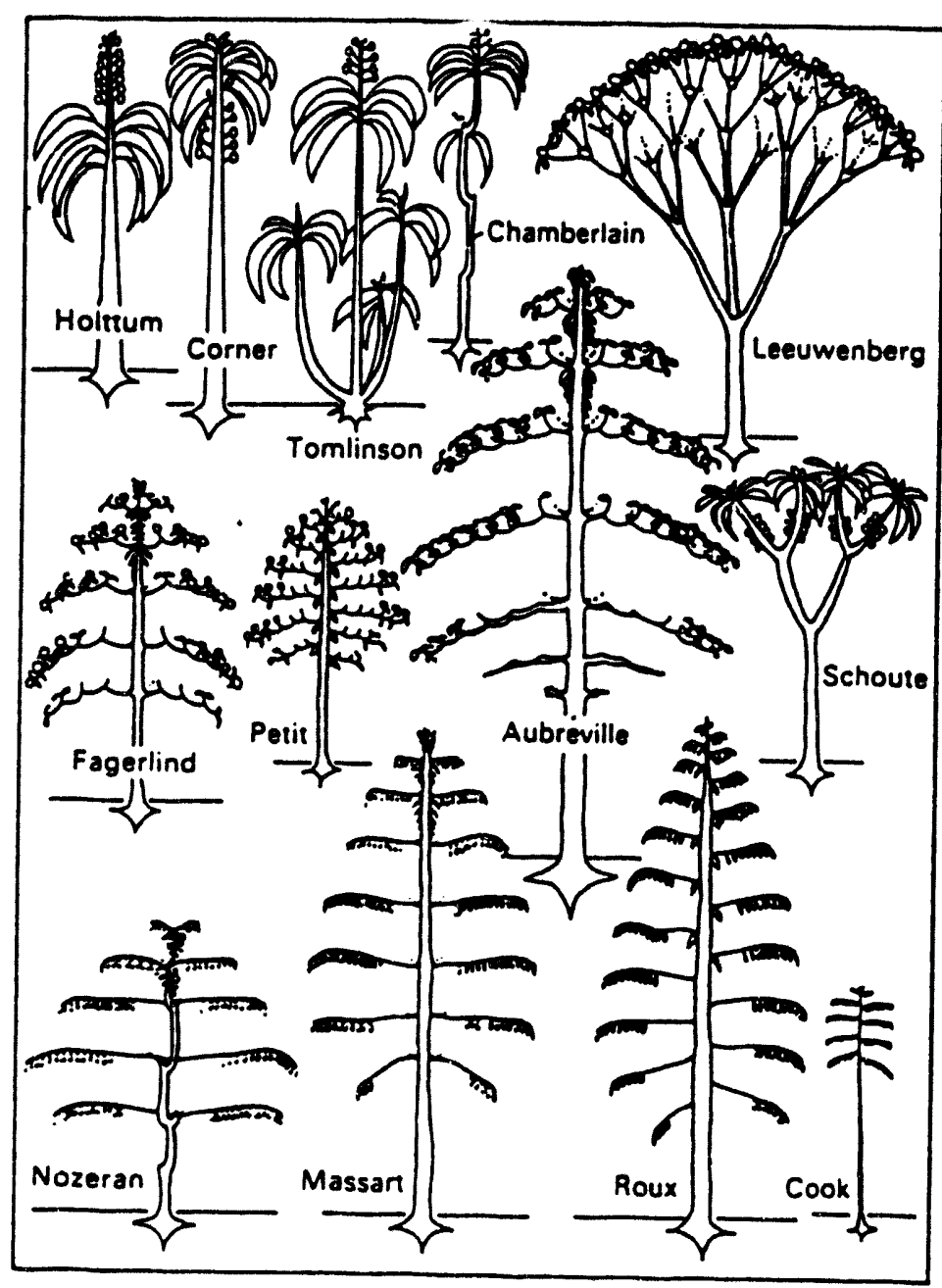

Figure 7. Developmental models of tree architecture, originally proposed by Halle and Oldeman (1970). (From Tomlinson, 1978). 


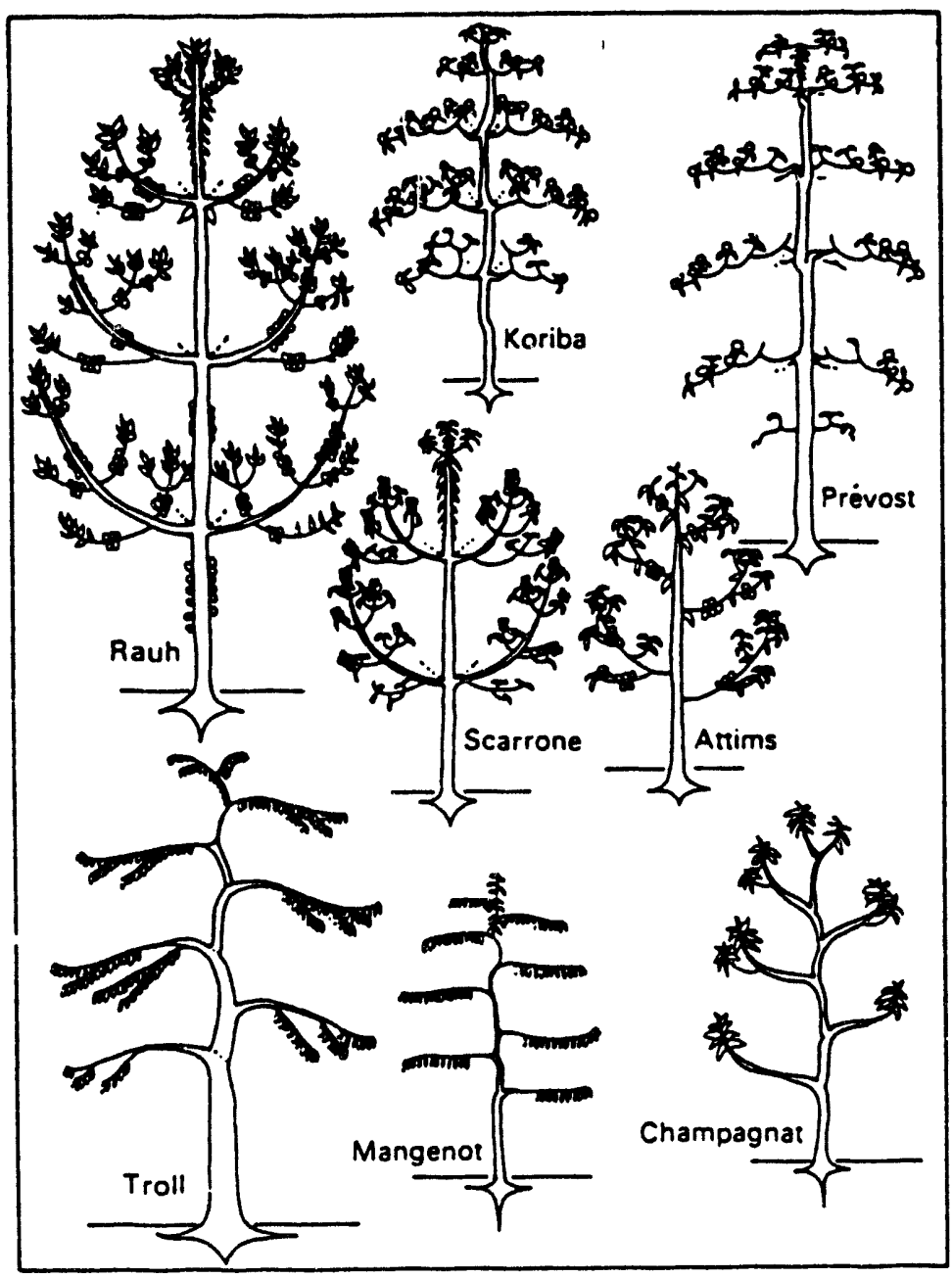


theory) that include the terminal leader, flower placement, branch origination mode, and other factors not found in the other schemes. The descriptive variables are assumed to have some explanatory content, influencing how the tree grows.

Both of these tree form classifications are entirely qualitative. We cannot match a given tree statistically to a particular ideotype or tree architectural form. Such testing would require a great deal of elaboration. Neither classification is a fully testable hypothetico-deductive theory, and, again, much elaboration would be required to reach that point. Nevertheless, such classifications are useful starting places for the development of theory.

\section{Food Webs}

Food webs provide a case that is more abstract than the tree branching case. Whereas vegetation has an obvious physical morphology, animal communities in general do not. The study of the interactions of animals has thus naturally focused on feeding relationships. A characterization of who eats whom and how much is called a food web.

The early realization that food webs could be graphically depicted and their energy flow quantified (e.g., Fig. 8) led to the perception that an important pattern of nature had been found. One of the earliest webs was presented by H.T. Odum for Silver Springs, Florida (Odum, 1957). This web showed not only trophic transfers of energy but also energy import and export via flowing water. The success of such studies led to the proliferation of food web data sets, comparisons between food webs in different habitats, and 


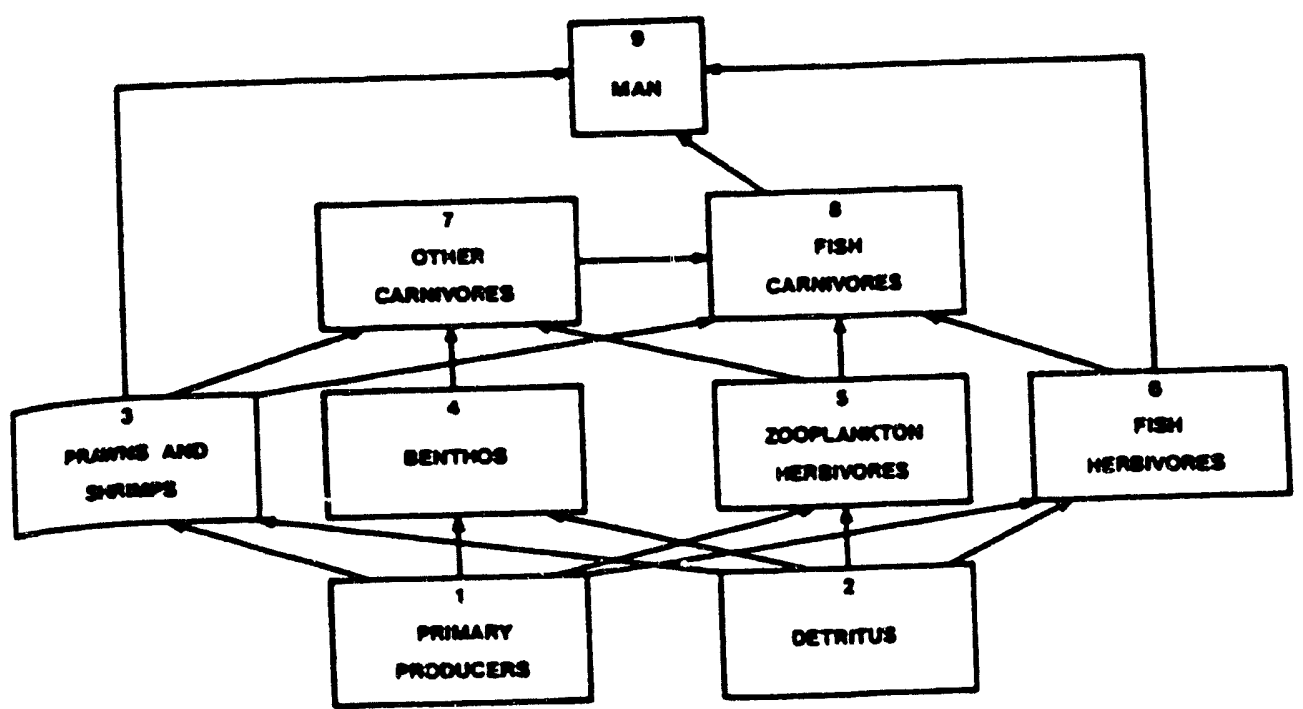

\.

Figure 8. Example typical food web. (From Yodzis, 1982). 
theoretical speculation concerning the causes of food web properties (e.g., Briand and Cohen, 1987; Cohen and Newman, 1988; Hastings, 1988; Kenny and Loehle, 1991; Kitching, 1987; Paine, 1988; Pimm and Kitching, 1987, 1988; Pimm and Rice, 1987; Sprules and Bowerman, 1988; Sugihara et al., 1989; Winemiller, 1989). Here, empirical patterns of apparent great informational content have thus provided the basis for experimental and theoretical studies aimed at explaining these patterns. Note that only the gross features of food webs were ever predicted or explained a priori, for example energy must be lost at each transfer between trophic levels because of thermodynamic considerations. Otherwise this is again a complex empirical pattern not discovered by a statistical test or an experiment.

The patterns detected in food webs also fail to match the key assumptions of statistical analysis that the objects or variables in the model are discrete, clear cut, or well defined. The pattern has shifted and changed throughout stage 2 (elaboration of the pattern), as have the measurement methods. For example, in attempting to relate food web structure to community dynamics, Paine (1980) showed that different methods of measuring the food web la qualitative web ( 0 or 1 connections) versus an energy flow web versus an influence web (who influences what other populations)] give very different pictures (Fig. 9). When a detailed treatment of a food web is attempted, the pattern that results (Fig. 10) is so complex that it challenges our belief (based on representations like Fig. 8) that we understand food web structure at all.

Kenny and Loehle (1991) proposed that connectance properties of food webs can be matched by a random connectance model modified by a sampling 
$10)^{\circ}$

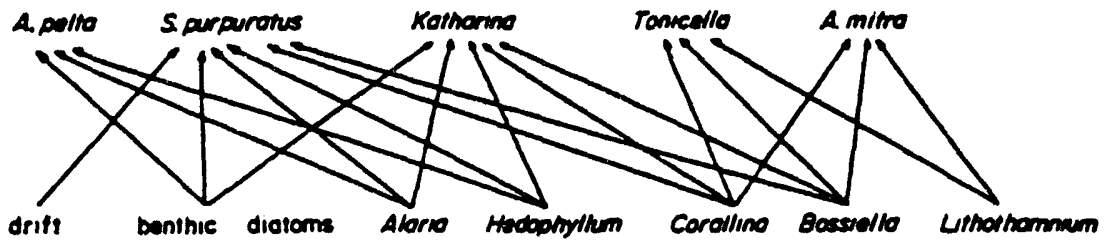

(b)

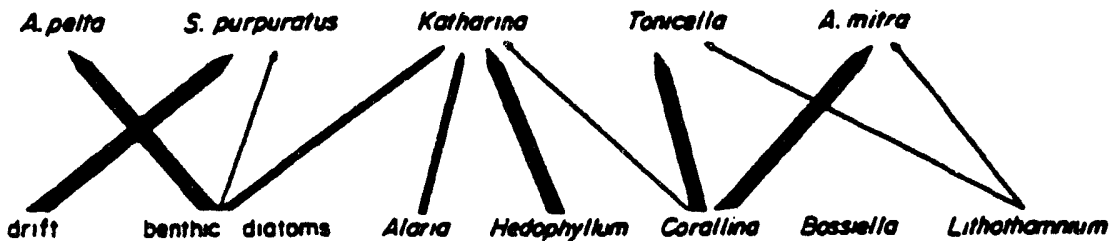

(c)

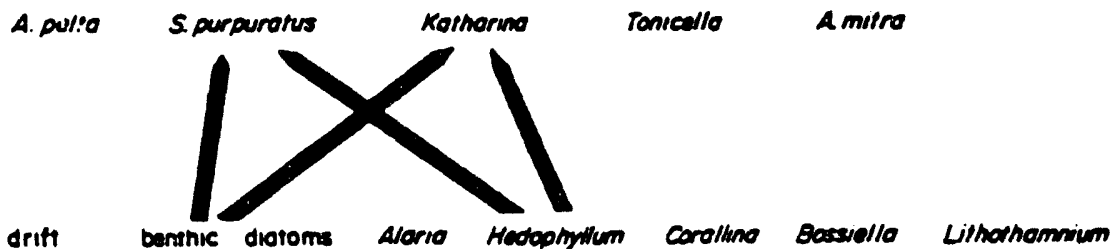

Figure 9. Comparison of three approaches to trophic relationships: A) qualitative connectedness, B) energy flow basis, C) influence relations based on controlled manipulation. (From Paine, 1980). 


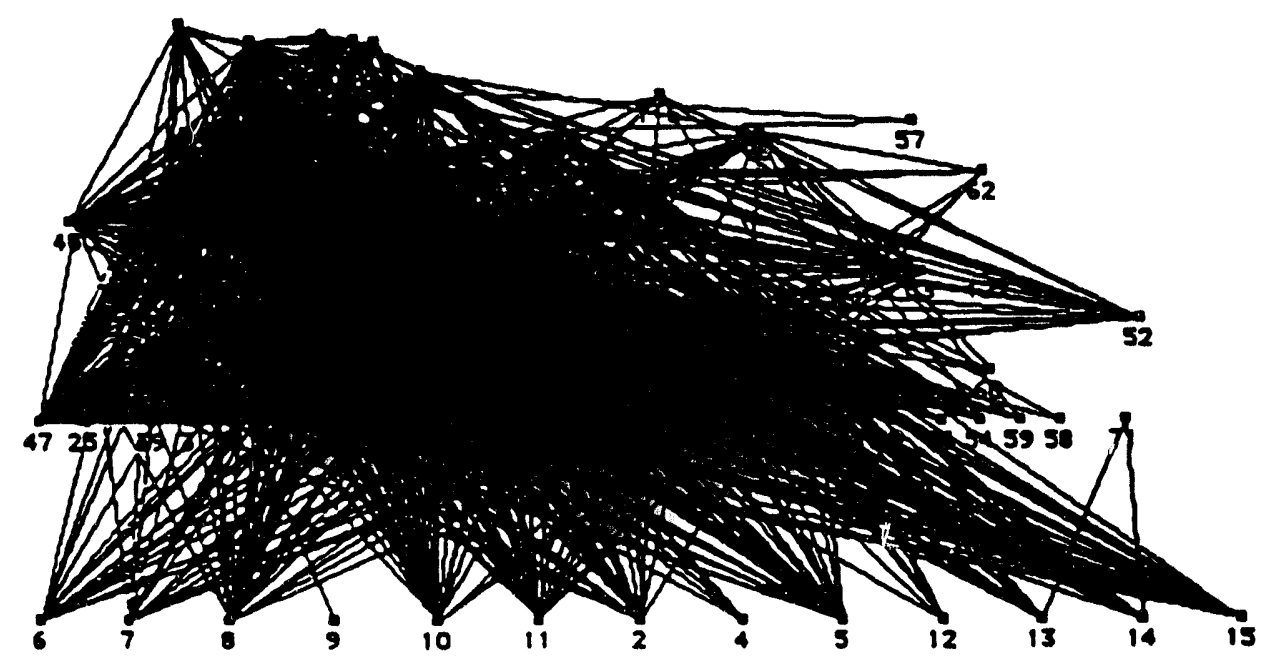

Figure 10. Food web complexity evident when trophic continuum approach applied (from Winemiller, 1989). Food web is for aquatic system at Caño Maraca, Venezuela. 
bias against small magnitude linkages, pointing out that the perception of pattern in food web data might in fact be an artifact. In their analysis, the most consistent patterns were found when respiration was specifically counted as a food web link for each species, pointing out the fluidity of our definition of "objects" in a food web. Thus, the key assumption that the patterns found for food webs relate in some simple way to the determination of community structure or conversely are the end product of certain organizing forces has been called into question. The pattern found in this case, food web structure, is much more a product of measurement methods and entity definition than were the tree crown patterns, because food webs are abstract rather than physical patterns. The pattern elaboration process is much more important here and involves issues such as changes with season, boundary definition, taxonomic resolution, measurement methods, and null models. Thus, at the moment food webs are rather like a Rorschach ink blot: Some complex pattern appears to be present, but what it is and whether it has meaning is less clear. The difficulty is that the mind is compelled to seek pattern, and to be satisfied when one is found. The danger in the case of food webs is that their visual presentation yields the appearance of a real pattern, but until we move away from diagrams and can rigorously and quantitatively compare them we do not know if the patterns are real.

\section{CONCLUSION}

The human brain not only recognizes patterns, but it seeks them out and will generate them even when they do not exist, as in clouds or ink blots. We may characterize much of science as the identification of complex natural patterns; their elaboration, quantification, and explanation; and, finally and 
definitely last, their statistical testing. The admonition that science requires good experimental design with replications within treatments is the death knell of discovery, largely limiting the practitioner to questions such as "the effect of levels of $X$ on $X . "$ Clinical trials, drug safety testing, agricultural research, and other fields are held in a death grip by this formalism. Complex patterns do not fit into an analysis of variance. In fact, long after the discovery of a pattern we may have only the vaguest idea of what we are measuring, how to describe it, or what the pattern means.

Another implication of the present argument concerns the lack of progress evident in certain fields of science. Bauer (1992) notes, for example, that in the social sciences conflicting theories and schools of thought exist without any resolution in favor of one or the other for indefinite periods of time. He attributes this problem to the ineffectiveness of the "reality therapy" that other sciences must endure. I would specifically argue that lack of progress in such fields is due to failure of the elaboration step. As Hall (1992) and Crease (1992) pointed out, technique is a crucial aspect of scientific progress because it allows us to measure things such that hypotheses become testable. In the social sciences there is no shortage of "scientific method" or hypotheses or advanced statistics (Bauer, 1992), rather what is missing is the capacity to reliably measure things or to define objects which are measurable. The type of elaboration of technique described by Hall (1992) and Crease (1992) often stops at the preliminary stage in the social sciences. Concepts are proposed which might be important or causative, but the further steps of elaboration are usually not possible. How do we reliably measure the Id, alienation, social unrest, happiness, or culture? Such terms illustrate the fallacy of reification, that just because one can use a word that therefore the 
word refers to a thing that exists. Instruments such as questionnaires and tests have been developed, but it is not clear what they actually measure. For example, an IQ test measures something that predicts success in school, but it is not clear that this something is "intelligence." Theories in the social sciences tend to be built of such concepts that we seem to understand but can not precisely quantify, and as such are subject to multiple interpretations. This interferes with rigorous testing against reality. This is not to say that true insights are not obtained in these fields, but rather that it is impossible to eliminate beliefs that do not accord with reality because tests that are acceptable to all parties as a proof cannot be obtained when the basic concepts are vague.

We should not underestimate the danger posed by the myths of the scientific method. The treatments of the Gallo and Baltimore fraud cases suggest that the auditors expected the type of rigor from these studies that might be found in a bank ledger, assuming that the scientists were (or should have been) following a set procedure. In reality, scientists on the cutting edge are struggling to measure things that no one understands, using methods that no one can prove are right. Aside from outright fabrication, the types of errors being criticized in the Gallo and Baltimore cases can be found in any laboratory where really innovative work is being done. Clarity, certainty, and well-defined lab protocols become available only long after the discovery phase. During the early days of the AIDS epidemic, for example, there was a complete muddle concerning symptoms and causation. Similar confusion exists now concerning chronic fatigue syndrome. In the case of AIDS, measuring the level of the HIV virus and correlating that to the degree of symptom expression gives a negative result because of the long latency of the 
virus and its odd behavior. By "proper" experimental design, therefore, we should reject HIV as causative.

Much confusion and misdirection results from the mistaken idea that first and foremost science is about doing controlled experiments with statistical rigor. In fact, science is largely about the struggle to convert an intuitively perceived pattern into something sharp and definite. By the time experiments are feasible and standard statistics apply, the battle is mostly won.

\section{ACKNOWLEDGEMENTS}

This work was not funded by Argonne National Laboratory. Thanks to Egolfs Bakuzis, Henry H. Bauer, Dan Herms, Rolfe Leary, and Mike Miller for helpful reviews and Karen Haugen for editorial assistance. 


\section{LITERATURE CITED}

Agu, M., and Y. Yokoi. 1985. A stochastic description of branching structures of trees. J. theor. Biology 112: 667-676.

Bauer, H.H. 1992. Scientific Literacy and the Myth of the Scientific Method. U. III. Press, Urbana.

Borchert, R, and N.A. Slade. 1981. Bifurcation ratios and the adaptive geometry of trees. Botanical Gazette 142:394-401.

Briand, F., and J.E. Cohen. 1987. Environmental correlates of food chain length. Science 238:956-960.

Brunig, E.F. 1976. Tree forms in relation to environmental conditions: An ecological viewpoint. In: M.G.R. Cannell and F.T. Last (eds.). Tree Physiology and Yield Improvement. Academic Press, London.

Cohen, J.E., and C.M. Newman. 1988. Dynamic basis of food web organization. Ecology 69:1655-1664.

Crease, R. P. 1992. The trajectory of techniques: Lessons from the past. Science 257:350-353.

Gleick, J. 1987a. Chaos: Making a New Science. Penguin Books, N.Y.

Gleick, J. 1987b. New images of chaos that are stirring a science revolution. Smithsonian, Dec. 1987:122-135.

Grebogi, C., E. Ott, and J.A. Yorke. 1987. Chaos, strange attractors, and fractal basin boundaries. Science 238:632-638.

Hadamard, J. 1945. The Psychology of Invention in the Mathematical Field. Princeton U. Press, Princeton, N.J. 
Hall, S. S. 1992. How technique is changing science. Science 257:344-349.

Halle', F. and R.A.A. Oldeman. 1970. Essai sur l'architecture et dynamique de croissance des arbres tropicaux. Masson et Cle, Paris.

Hastings, A. 1988. Food web theory and stability. Ecology 69:1665-1668.

Kenny, D. and C. Loehle. 1991. Are food webs randomly connected? Ecology 72:1794-1799.

Kitching, R.L. 1987. Spatial and temporal variation in food webs in waterfilled treeholes. Oikos 48:280-288.

Lightman, A. and O. Gingerich. 1992. When do anomalies begin? Science 255:690-695.

Loehle, C. 1988. Philosophical Tools: Potential Applications to Ecology. Oikos 51:97-104.

Margolis, H. 1987. Patterns, Thinking, and Cognition. Univ. of Chicago Press, Chicago.

Odum, H.T. 1957. Trophic structure and productivity of Silver Springs, Florida. Ecol. Monographs 27:55-112.

Paine, R.T. 1980. Food webs: Linkage, interaction strength and community infrastructure. J. Animal Ecology 49:667-685.

Paine, R.T. 1988. Food webs: Road maps of interactions or grist for theoretical development? Ecology 69:1648-1654.

Pimm, S.L. and R.L. Kitching. 1987. The determinants of food chain lengths. Oikos 50:302-307.

Pimm, S.L. and R.L. Kitching. 1988. Food web patterns: Trivial flaws or the basis of an active research program? Ecology 69:1669-1672 
Pimm, S.L. and J.C. Rice. 1987. The dynamics of multispecies, mult-life-stage models of aquatic food webs. Theoretical Population Biology 32:303-325.

Popper, K.R. 1963. Conjectures and Refutations: The Growth of Scientific Knowledge. Harper \& Row, N. Y.

Root-Bernstein, R.S. 1989. Discovering. Harvard U. Press, Cambridge.

Shinozaki, K., K. Yoda, K. Hozumi, and T. Kira. 1964. A quantitative analysis of plant form - the pipe model theory. II. Further evidence of the theory and its application in forest ecology. Japanese J. Ecology 14:133-139.

Sidorowich, J.J. 1992. Repellors attract attention. Nature 355:584-585.

Sprules, W.G., And J.E. Bowerman. 1988. Omnivory and food chain length in zooplankton food webs. Ecology 69:418-426.

Sugihara, G., K. Schoenly, and A. Trombla. 1989. Scale invariance in food web properties. Science 245:48-50.

Tomlinson, P.B. 1978. Branching and axis differentiation in tropical trees. pp. 187-208 in: P.B. Tomlinson and M.H. Zimmerman (eds). Tropical Trees as Living Systems. Cambridge University Press, N.Y.

Waller, D.M. and D.A. Steingraeber. 1985. Branching and modular growth: Theoretical models and empirical patterns. pp. 225-257 in: J.B.C. Jackson, L.W. Buss and R.E. Cook (eds.). Population Biology and Evolution of Clonal Organisms. Yale U. Press, New Haven.

Winemiller, K.O. 1989. Must connectance decrease with species richness? American Niaturalist 134:960-968.

Yodzis, P. 1982. The compartmentation of real ana assembled ecosystems. American Naturalist 120:551-570.

Watson, J.D. 1968. The Double Helix. New American Library, N.Y. 
11
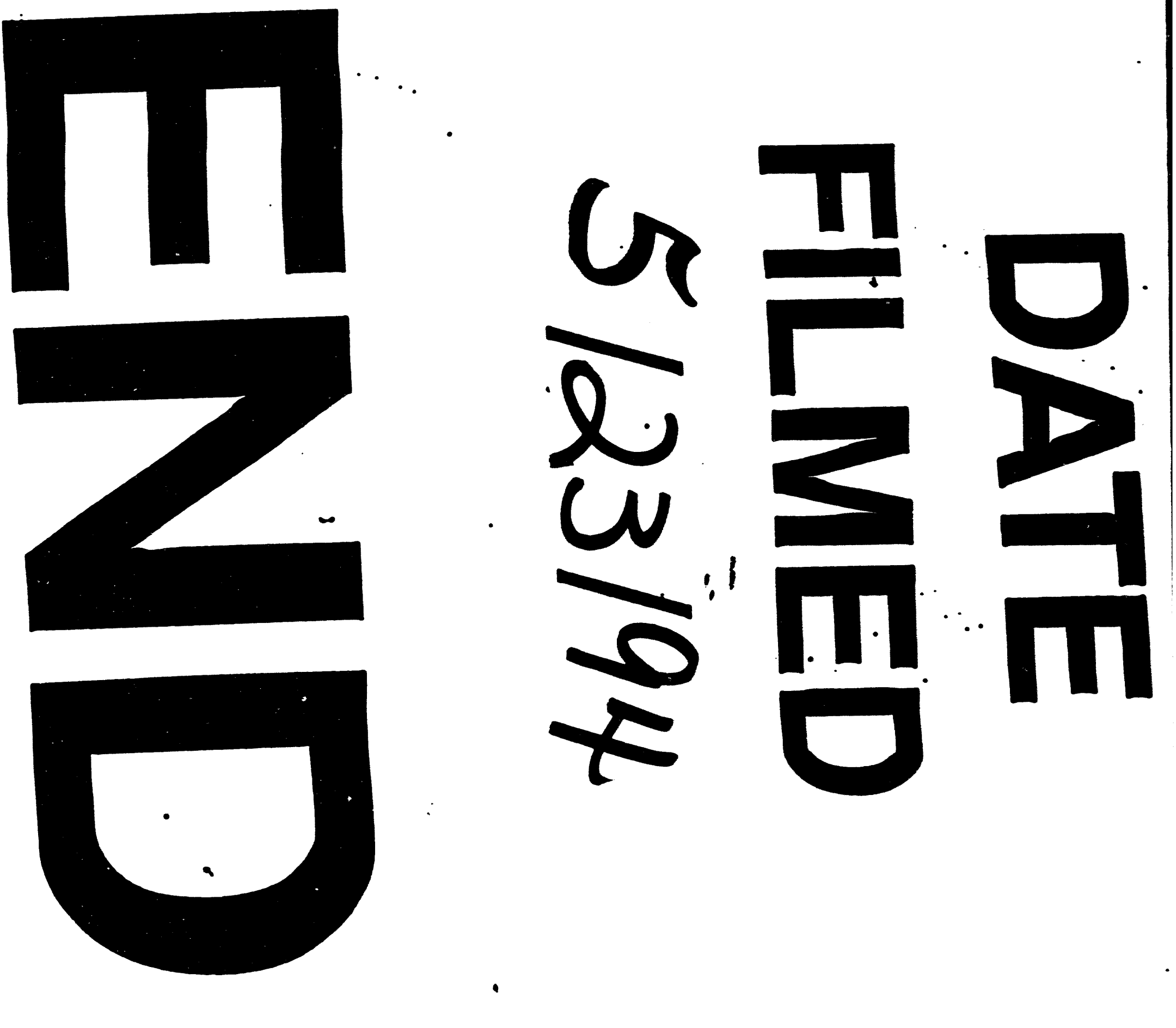
$$
\text { L }
$$

$\checkmark$ 